\title{
A Cross-Sectional Survey on Knowledge, Attitudes and Practices Towards Malaria Control and Prevention Among Caregivers of Children Under-5 in the Western Area of Sierra Leone
}

\author{
Joan Mabinty Koroma \\ Southern Medical University \\ Yuji Wang \\ Southern Medical University \\ Xiang Guo \\ Southern Medical University \\ Xiaoqing Zhang \\ Southern Medical University \\ Jone Jama Kpanda Ngobeh \\ Southern Medical University \\ Ahmed Mohamed Elamin Ali Gabir \\ Southern Medical University \\ Ziyao Li
}

Southern Medical University

Li Li

Southern Medical University

\section{Rangke Wu}

Southern Medical University

Xiaohong Zhou ( $\nabla$ daizhouxh@163.com )

Southern Medical University https://orcid.org/0000-0003-4237-5436

\section{Research}

Keywords: Malaria, Sierra Leone, Knowledge, Attitudes, and Practices (KAPs), Caregivers, Children under5 , Community intervention

Posted Date: March 6th, 2021

DOI: https://doi.org/10.21203/rs.3.rs-270948/v1 
License: (c) (i) This work is licensed under a Creative Commons Attribution 4.0 International License. Read Full License 


\section{Abstract}

\section{Background}

Children under- 5 are the most vulnerable to malaria infection and they suffer serious complications. Sierra Leone is one of the countries with the highest malaria burden in the world. This study aimed to assess the knowledge, attitudes and practices (KAPs) towards malaria control and prevention among caregivers of children under- 5 in the Western Area of Sierra Leone.

\section{Methods}

A cross-sectional survey was conducted among caregivers of children under-5 visiting the out-patient department of six selected hospitals/community health centers. Data were collected via questionnaire interview with 350 caregivers. Further statistical analyses were performed primarily Spearman's rank test for inferring the correlations among KAPs, univariate and multivariate logistic regression for demonstration the association between KAPs and socio-demographic characteristics.

Results

Women made up $97.1 \%$ of respondents with the majority being young mothers-including 33 single student moms. $46.3 \%$ among respondents were unemployed and $27.1 \%$ had no educational background. Only $1.4 \%$ received malaria related information from the internet/social media. This KAPs survey indicated that misconception of cause, transmission and clinical symptoms of malaria; unawareness of lethality and severity; inappropriate behaviors in prevention and treatment such as self-medicating were still in existence in caregivers. But a positive correlation in knowledge-attitudes $\left(r_{s}=0.13, P<0.05\right)$ and attitudes-practices $\left(r_{s}=0.45, P<0.001\right)$ were revealed. The caregivers being a mother and having at least secondary education were demonstrated associated with positive attitudes and practices. Meanwhile, more urban-based caregivers (79.8\%) followed a complete malaria treatment course of ACTs than the rural (63.3\%), but in view of ITNs use, more rural-based caregivers presented positive attitudes (85.3\%) and practices $(70.1 \%)$ than the urban $(69.9 \%, 52.0 \%)$. The student moms might know more but do less.

\section{Conclusions}

In order to better protect children under- 5 against lethal malaria, their caregivers, especially young mothers, need strong support to reduce misconceptions, inappropriate behaviors, and provide increased vigilance. It is important to develop an efficient communication channel to disseminate information regarding malaria, especially strengthening community-based media infrastructure, including internet/social media. A increase in education and employment opportunities for women, as well as setting up community-based malaria counselling services, is urgently needed.

\section{Background}


Malaria spans in 109 countries across all continents and its occurrences and distributions vary with both different geographic and climate conditions, and the social, cultural and hygienic habits of the population, but is mostly concentrated in the underdeveloped tropical and subtropical regions worldwide $[1,2]$. Among the estimated 405,000 deaths resulted from estimated 228 million cases of malaria in 2018 , nearly $85 \%$ of them were reported in 20 countries in the WHO African Region and India, among which Sierra Leone bore a heavy malaria burden with 2,451,110 cases and 6,564 deaths that year [1]. Malaria is among the top 10 causes of death globally, with an estimated $70 \%$ of all malaria deaths occurring in children under-5, mostly in Sub-Saharan Africa (SSA). In Sierra Leone, the entire population is at risk of malaria, and children under- 5 and pregnant women are the most vulnerable groups. Etiologically, malaria is mainly transmitted through the bites of the female Anopheles mosquitoes infected with Plasmodium falciparum which is responsible for over $90 \%$ of malaria cases and all the severe forms of the disease in Sierra Leone $[1,3]$.

In postwar and post-Ebola Sierra Leone, malaria control efforts face the inadequate capacity due to its weak health care system, the poor hygienic practices of people, and other poverty-stricken social and environmental condition. Especially in remote rural areas and swamp regions, people are less accessible to health facilities and highly subjected to malaria devastation consequently [4]. Although malaria is curable using Artemisinin-based Combination Therapies (ACTs), the prevention and control of this highly transmissible disease still places an increased challenge on public health systems at global, national, subnational and community levels, and poses a huge health burdens on both the government and individuals, hindering the socioeconomic development and even leading to deeper poverty [5]. Furthermore, severe malaria impairs children's learning and cognitive abilities by as much as $60 \%$, consequently affecting the performance of Sierra Leone's primary and secondary education programs [6].

Over the past several years, Sierra Leone has made progress to control the impact of malaria among the citizens. Such progress has included in the development of a National Malaria Control Programme (NMCP) Strategic Plan 2016-2020 and the revision of guidelines in December 2015 to ensure that the programme is implemented on the evidence basis $[1,6]$.

However, incomplete knowledge and misconceptions about the disease still exist and probably hinder correct practices individuals should take towards malaria prevention, such as increased exposure to the disease, inappropriate implementation of interventions, inadequate recognition of the factors that contribute to the occurence and development of disease, thus creating an obstacle to effective malaria control. This is evidenced by a high rate of outpatient-clinic visits for malaria accounting for about $47 \%$ of outpatient morbidity for under five children, and $37.6 \%$ of all hospitalizations with a case fatality of $17.6 \%[6]$.

Researches [7-10] concerning individual knowledge, attitudes and practices (KAPs) have shown that factors, including education levels, in some instances, are related to behaviour in malaria prevention and control. Behaviour is an important component in malaria prevention and control, but the behavioral status against malaria affected by the KAPs levels in the community was even more crucial [11]. 
Primary caregivers play key roles in the health care to children under-5, being responsible for a majority of the everyday practices and decisions that determine the child's health status. A mother/caregiver's knowledge about the disease, and their attitudes and practices towards protecting their children from malaria are essential for seeking appropriate medical care in the case of illness, and administering recommended treatments [12]. Thus, an insight into the KAPs of caregivers towards malaria may contribute to the evidence-based malaria prevention and control, as well as the well-informed design of community interventions in order to reduce the prevalence of malaria in children under-5.

\section{Methods}

Setting: This study was carried out in the Western Area, one of four principal divisions of Sierra Leone. The Western Area comprises the oldest city and national capital Freetown and its surrounding towns and countryside and it covers an area of $557 \mathrm{~km}^{2}$ with a total population of about 1.5 million people (2015 National census). The Western Area is divided into two districts: Western Area Rural (WAR) and Western Area Urban (WAU).

This study was carried out in six selected health care facilities in two districts-WAU and WAR (Fig. 1), three from WAU: Ola During Children's Hospital (ODCH) $\left(8^{\circ} 29^{\prime} 25^{\prime \prime} \mathrm{E}, 13^{\circ} 13^{\prime} 7 " \mathrm{~S}\right)$, which is the biggest

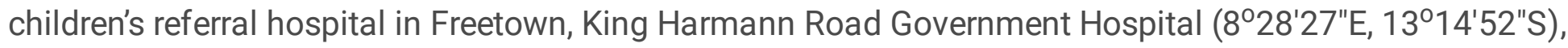
and Jenner Wright Hospital $\left(8^{\circ} 29^{\prime} 7^{\prime \prime} \mathrm{E}, 13^{\circ} 12^{\prime} 43^{\prime \prime} \mathrm{S}\right)$; and another three from WAR: Waterloo Community

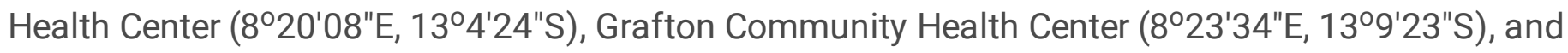
Lakka Community Health Center $\left(8^{\circ} 23^{\prime} 49^{\prime \prime} \mathrm{E}, 13^{\circ} 15^{\prime} 50^{\prime \prime} \mathrm{S}\right)$. These six hospitals or centers were selected because they are in strategic locations accessible for most people in the communities without much difficulty. Since they are all government-owned and offers free health care services for children under- 5 , these facilities almost always have a large number of caregivers for children under- 5 accessing their healthcare services.

Study population and eligibility criteria: Caregivers with one or more child(ren) under-5 residing in WAR and WAU who visited the out-patient departments of the selected health care facilities were included.

\section{Sample size:}

The sample size was calculated using the Cochran's formula (1977) [13] 


$$
n=\frac{z^{2}[(p) \times(1-p)]}{d^{2}}
$$

$$
n=\frac{1.96^{2} \times 0.205 \times(1-0.205)}{0.05^{2}}
$$

$$
n=250
$$

Where: $\mathrm{n}=$ sample size; $\mathrm{z}=\mathrm{z}$ value 1.96 for $95 \%$ confidence level; $p=$ proportion of malaria prevalence in children under- 5 in the Western Area Urban and Rural Districts of Sierra Leone, according to the 2016 National Malaria Indicator Survey $(20.5 \%=0.205)[14]$; and $d=$ absolute error allowance/precision (0.05).

$40 \%$ (100) of the calculated sample size was added to the study population to account for missing vital data, respondents partially answering questions or withdrawal during the interview, or incomplete questionnaire. Hence, the final total sample size was $(250+100)=350$.

Survey questionnaire: A questionnaire was developed under our conceptual framework and referring to previous studies $[15,16]$, which contained open and close-ended questions and was divided into four sections (Additional file 1). Section A focused on the socio-demographic characteristics of the caregivers such as age, sex, religion, relationship with the under- 5 child(ren) they take care of, number of children under-5 and above-5, districts (urban/rural), marital status, educational level and occupational status. Section B assessed the caregiver's knowledge about the cause of malaria and its mode of transmission, signs and symptoms of malaria, and prevention. Section $C$ assessed the caregiver's attitudes towards malaria such as how serious of a health problem they consider malaria to be, if they sleep under a bed net with their child(ren), what time of day they think mosquitoes bite most, and what they think is the best treatment for malaria. Meanwhile, section D assessed the caregiver's practices towards malaria prevention such as, the kind of measures they take to protect their children from mosquito bites, what they do when their child(ren) has a fever or is prescribed an antimalaria medication, what influences their action and how long they wait before seeking medical care for their febrile child(ren).

Statistical analysis: Socio-demographic characteristics and KAPs data were collected via the questionnaire interview method from 350 caregivers. Descriptive statistics was used to analyze the sociodemographic characteristics of the respondents, and the results were expressed in frequencies and percentages. A scoring system was utilized in the evaluation of the KAPs data (Additional file 2). Two KAPs levels were defined by the $60 \%$ cut-off value of total score. The responses to the KAPs variables between the respondents from WAU and WAR were compared using Chi-square analysis. Fisher's exact test was used when more than $20 \%$ of cell counts less than five. Spearman's rank test was used to 
determine the extent of correlation between KAPs scores since data was not normally distributed as per outcome of the Kolmogorov-Smirnov test. Based on the rule of thumb of Cohen [17], the strength of correlation was interpreted as $0=$ no relationship, $0.10-0.29=$ small $/$ low correlation, $0.30-0.49=$ medium/moderate correlation, and $0.50-1.00=$ large/high correlation .

The association between KAPs and the socio-demographic characteristics of the respondents was evaluated by univariate analyses. All variables significant in the univariate analysis with a $P \leq 0.25$ were included in the multivariate logistic regression model. Reduced subset models were developed using elimination based on the AIC (Akaike Information Criteria) score. The level of statistical significance was set at 0.05 . All of the data analyses and figure drawing were conducted in R software (Version: 3.6.1).

\section{Results}

\section{Socio-demographic characteristics of the caregivers}

As shown in Table 1, all 350 caregivers interviewed completed the survey with a nearly equal number of respondents from WAU and WAR. Most of them were less than 30 year-old mothers. $70.0 \%$ of caregivers were married, while $19.4 \%$ were single, and most caregivers were unemployed younger mothers. The total unemployed reached $46.3 \%$ including 33 younger students. $27.1 \%$ of them had no educational experience and only $64.3 \%$ had at least a secondary level of education. But all of them had at least one child under-5 living with them and $54.6 \%$ had at least one more child above- 5 .

\section{Knowledge}

$99.1 \%$ of the caregivers had heard about malaria, and no significant difference of malaria related information sources $(P=0.858)$ was found between the caregivers from WAR and WAU (Additional file 3: Table S1). Most of them got malaria related knowledge from health workers/facilities (89.0\%), followed by radio $(60.8 \%)$, television (17.6\%) and printed materials such as billboards/handbills ( $8.1 \%)$. Besides, $26.8 \%$ got information from other sources such as the community sensitization and announcements about malaria, their friends/peers and also neighbors, and only $1.4 \%$ were informed through the internet/social media, even only $1.7 \%$ of those residing in WAU where the use of internet/social media was expected to be high, just a little higher than those living in WAR (1.1\%) (Fig. 2).

As displayed in Fig. 3 and Additional file 3: Table S1, the percent of respondents that did not know malaria can be prevented, cured and lead to death were $18.0 \%, 3.4 \%$, and $8.9 \%$, respectively. Among them, only $1.2 \%$ of urban-based caregivers were unaware of lethality of malaria, whereas the number for ruralbased caregivers was over four times higher $(5.6 \%)(P=0.05)$. When asked about the symptoms of malaria, the most common responses were fever $(71.1 \%)$, followed by vomiting ( $42.9 \%)$, loss of appetite (30.9\%), and body and joint pains (26.9\%), with only a few responses for headaches $(9.1 \%) .58 .6 \%$ of them stated other signs and symptoms they knew such as weakness and dizziness, dark colored urine, yellow/pale/white eyes, etc. Approximately $10.0 \%$ didn't know that malaria parasites can be transmitted through mosquito bites. This study revealed that, misconceptions about the causes and mode of 
transmission of malaria still prevailed among the caregivers, as $0.3 \%$ of them stated that eating too much and having close contacts with a person who has malaria can cause malaria. Also, $4.0 \%$ of them stated other misconceptions about the cause of malaria such as eating too many oranges, not washing hands regularly, cold and flu, dirty hands and feet, flies, drinking too many sweet/soft drinks, etc. While $86.6 \%$ of the caregivers stated that bushes/dirty places were convenient resting/breeding places for mosquitoes, followed by stagnant water (51.1\%), and dark places/sheds (11.7\%).

\section{Attitudes}

As summarized in Fig. 3 and Additional file 4: Table S2, although $90.0 \%$ of respondents believed that malaria is a very serious health problem, still 3.1\% said "No", 5.4\% "Not sure" and 1.4\% "Don't know". $77.7 \%$ said that they and their child(ren) do sleep under a bed net, while the rural-based caregivers(85.3\%) showed significantly more positive attitudes than the urban $(69.9 \%)(P<0.001)$. Explaining the lack of bed net for their children, $46.2 \%$ (46/78) of them mentioned that they could not afford it, $32.1 \%$ said not readily available, $6.4 \%$ did not like sleeping under a bed net, $3.8 \%$ do not think it's important, and $11.5 \%$ stated other reasons such as the statements that they and their child(ren) had allergic reactions or sweat a lot whenever they slept under a bed net (Additional file 4: Table S2, Fig. 4a). Moreover, when asked about how often your child(ren) used the bed net, $85.7 \%$ stated always and $14.3 \%$ sometimes, but $21.7 \%$ did not think the bed net was treated with insecticide. Meanwhile, $88.0 \%$ of the respondents thought that malaria-transmitted mosquitoes bite mostly at night. $65.1 \%$ stated that they think that Artemisinin-based Combination Therapies (ACTs) were the best treatment for malaria, but 32.0\% said "Don't know", and $1.4 \%$ also shared other opinions such as injections, intravenous solutions, etc.

\section{Practices}

As shown in Fig. 3 and Additional file 5: Table S3, although $77.1 \%$ of respondents would go to a hospital or clinic when their child(ren) had a fever, $19.4 \%$ still preferred self-medicating first at home, and $3.4 \%$ went first to a pharmacy. As stated by $93.4 \%$ of the caregivers, the most important factor influencing their action to seek medical help was the condition of the child, followed by perceived cost involved (3.7\%) and time availability (1.7\%). Furthermore, only $54.6 \%$ of the caregivers would seek medical attention for their febrile child(ren) within 24 hours, whilst $45.1 \%$ usually wait for 2-5 days. Meanwhile, when an antimalarial medication had been prescribed, only $71.4 \%$ of caregivers mentioned administration of a complete treatment course, but $12.9 \%$ stop administration as soon as the child began to show improvement. There was a significantly higher rate among the urban-based caregivers $(79.8 \%)$ who performed a complete treatment course than among the rural ones $(63.3 \%)(P<0.001)$.

Regarding the use of protective measures against malaria-transmitted mosquitoes, the most common practices employed by $61.1 \%$ of respondents slept under an insecticides treated nets (ITNs), the ruralbased caregivers had a significantly higher rate of ITNs use than the urban $(70.1 \%$ vs. $52.0 \%, P<0.01)$ (Fig. 4a). But we observed that misuse of bet nets such as using for doing pond fishing (Fig. 4b) and covering backyard gardens (Fig. 4c) is still common especially in rural and provincial areas of Sierra Leone. Other common selections for protection included measures of using mosquito repellant (40.9\%), 
regularly cleaning surroundings of the house (31.7\%), wearing protective clothing (25.1\%), and using insecticide spray (24.9\%). Only a few caregivers said that they got rid of stagnant water $(8.3 \%, 29 / 350)$, and cleared bushes around the house $(3.4 \%, 12 / 350)$, while $11(6.4 \%, 11 / 173)$ of them residing in WAU and only 1 from WAR $(0.6 \%, 1 / 177)$. Also, $6.0 \%$ of caregivers mentioned other protective measures such as closing the windows and doors early before dark, fixing a mesh on window and door frames to keep mosquitoes out, etc (Fig. 3-4, Additional file 5: Table S3).

\section{Correlations among knowledge, attitudes and practices}

Inferred by the spearman correlation test (Table 2), a significant positive correlation was found between knowledge-attitudes $\left(r_{s}=0.13, P<0.05\right)$ and attitudes-practices $\left(r_{s}=0.45, P<0.001\right)$. Further univariate logistic regression analysis indicated that improved knowledge might positively affect attitudes (OR = $1.98 ; 95 \% \mathrm{Cl}=1.21-3.25)$, while positive attitudes might lead to good practices $(\mathrm{OR}=7.91 ; 95 \% \mathrm{Cl}=4.33-$ 15.54).

\section{Effects of socio-demographic characteristics on KAPs}

Through univariate analysis, the association of socio-demographic and malaria-related KAPs were demonstrated by comparing with referred groups (Table 3 ). The age, religion, educational background and occupation were found to correlate with knowledge. The caregivers in the $>30$ year-old group tended to have higher knowledge than the $15-20$ year-old group $(\mathrm{OR}=3.03,95 \% \mathrm{Cl}=1.35-7.28)$. In comparison to the Muslim religion, Caregivers' Christian faith was positively associated with knowledge $(O R=2.20$, $95 \% \mathrm{Cl}=1.29-3.86)$. The caregivers with secondary education had higher knowledge than the group with no education $(\mathrm{OR}=1.94,95 \% \mathrm{Cl}=1.15-3.28)$. Meanwhile, comparing with the unemployed caregivers, students knew more malaria-related information $(\mathrm{OR}=3.04,95 \% \mathrm{Cl}=1.20-9.33)$, but represented a negative practice $(\mathrm{OR}=0.22,95 \% \mathrm{Cl}=0.07-0.56)$. As for attitudes, age, educational background and district (urban or rural) were inferred to be associated. A positive association with attitudes was found in the respondents with a university education $(\mathrm{OR}=4.46,95 \% \mathrm{Cl}=1.42-19.73)$, and the rural-based caregivers $(\mathrm{OR}=1.83,95 \% \mathrm{Cl}=1.14-2.96)$, but a negative association was found in the caregivers of the $21-25$ year-old group $(\mathrm{OR}=0.39,95 \% \mathrm{Cl}=0.19-0.76)$.

Furthermore, independent predictors of KAPs were inferred by a multivariate logistic regression analysis comparing with referred groups (Table 4). The age, religion, education and occupation were found to be independent predictors for malaria-related knowledge. The groups of $26-30$ year-old $(\mathrm{OR}=2.14,95 \% \mathrm{Cl}=$ $1.02-4.55)$ and $>30$ year-old $(\mathrm{OR}=4.83,95 \% \mathrm{Cl}=1.95-12.69)$, the caregivers with a secondary education $(\mathrm{OR}=2.34,95 \% \mathrm{Cl}=1.32-4.19)$, the Christians $(\mathrm{OR}=2.28,95 \% \mathrm{Cl}=1.30-4.14)$, and the students $(\mathrm{OR}=2.98$, $95 \% \mathrm{Cl}=1.12-9.48)$ were more likely to have a higher knowledge about malaria. Meanwhile, district, education, age and relationship were found as the independent predictors for malaria-related attitudes. The rural-based caregivers $(\mathrm{OR}=1.97,95 \% \mathrm{Cl}=1.20-3.28)$, the group with a higher education (university level, $\mathrm{OR}=5.53,95 \% \mathrm{Cl}=1.67-25.54$; secondary level, $\mathrm{OR}=1.86,95 \% \mathrm{Cl}=1.05-3.30$ ) were more likely to have a positive attitude towards malaria, while caregivers in the $21-25$ year-old group $(O R=0.40,95 \% \mathrm{Cl}=$ 
0.19-0.79) and fathers $(\mathrm{OR}=0.19,95 \% \mathrm{Cl}=0.04-0.80)$ were more likely to have negative malaria-related attitudes. Meanwhile the relationship, occupation, and marital status were taken as independent predictors for malaria-related practices. The mothers seemed more likely to have better practices towards malaria control and prevention as compared to the fathers $(\mathrm{OR}=0.19,95 \% \mathrm{Cl}=0.04-0.80)$. The caregivers who were students $(\mathrm{OR}=0.11,95 \% \mathrm{Cl}=0.01-0.55)$ or married $(\mathrm{OR}=0.53,95 \% \mathrm{Cl}=0.29-0.97)$ or in a consensual union $(\mathrm{OR}=0.30,95 \% \mathrm{Cl}=0.12-0.74)$ were more likely to have bad malaria-related practices.

\section{Discussion}

\section{Knowledge}

Similar to previous studies $[8,15]$, the respondents in the Western Area in Sierra Leone received malaria related information mainly from health workers/facilities followed by radio. While $26.6 \%$ of respondents named other sources of information such as community sensitization, meetings and announcements, in accordance with the study conducted in Swaziland [8]. Only a few caregivers mentioned TV and printed materials (billboards/handbills, etc) as sources, even fewer got the information from the internet or social media, which might be due to the low usage and high cost of internet technologies in Sierra Leone. But in Saudi Arabia, the main source of malaria related information was recorded from the internet and social media [9]. Therefore, in Sierra Leone, more attention should be given to enhance caregivers' knowledge by continuously improving the content, type, and media of malaria related messages, especially strengthening the media infrastructure of the communities such as the internet or social media, TV and radio, in order to achieve proper health education coverage for various groups. Moreover, using simple audio-visual messages in local languages will help develop a highly effective means of communication especially for the caregivers who are not or under-educated.

For the knowledge about symptoms of malaria, fever was the highest known symptom followed by other symptoms such as weakness, dizziness, dark colored urine, etc which were mostly mentioned by the respondents. Fever has also been recorded as the most known and common symptom of malaria in children under-5 in Cabo Verde and Zambia[2, 10]. Other symptoms such as anemia were most associated with malaria in children under-5 according to reports in Tanzania [22, 23]. Similar to other surveys $[24,26]$, our study revealed that about $90 \%$ of respondents know malaria transmitted through the bite of infected mosquitos. However, some misconceptions about the cause and transmission of malaria were mentioned by some caregivers such as eating too many oranges, not washing hands regularly, catching a cold and flu, dirty hands and feet, flies, drinking too many sweet/soft drinks, etc. The other misconceptions of malaria transmission which included being exposed directly to the sun for a long time, drinking dirty water, etc, were also reported in Tanzania and Uganda [22, 27]. Regarding the resting and breeding places of mosquitoes, bushes/dirty places and stagnant water were the most mentioned by respondents which is consistent with previous study in Tanzania [28].

\section{Attitudes}


In the present study, malaria is known as a very serious health problem by $90 \%$ of caregivers, similar to previous surveys in south-western Saudi Arabia and Rwanda [32,33]. 88\% of the respondents stated that mosquitoes bite mostly at night time. This kind of attitude is expected to influence caregivers to be more vigilant especially at night in protecting their children from mosquito bites, but most importantly to get rid of mosquito breeding sites and sleep under an ITN. Recent studies $[37,38]$ have corroborated the idea about the time-of-day of blood-feeding and malaria transmission by mosquitoes to be mostly at night time. Compared with an earlier report of Sierra Leone Demographic Health Survey (SLDHS) in 2013 recorded that only $49.5 \%$ of children under-5 slept under a bed net at night [34], our findings showed that $77.7 \%$ of respondents together with their children slept under a bed net. But there were still 78 caregivers who did not use the bed net, 46 among them stated that they could not afford it. These are most likely individuals who are socioeconomically disadvantaged and are probably waiting on usual mass mosquito nets donation campaigns to get one, as some even mentioned that they were not supplied a bed net at their community health centers, or claimed that mosquito nets are not readily available, which means they could probably afford it but cannot easily find one for purchase. In essence, some of these common barriers to the use of bed nets have also been discovered by other studies [35, 36]. Moreover, $21.7 \%$ of caregivers still did not know of ITNs. Meanwhile, ACTs are recommended by the WHO, and are provided by the health facilities of governments and even sold in pharmacies and drug stores nationwide as a part of the first-line anti-malaria treatment policy in Sierra Leone $[39,40]$. But $32 \%$ of caregivers said that they did not know ACTs are the best treatment for malaria.

\section{Practices}

Every respondent in this study stated that they employed at least one method of prevention against mosquito bites with the highest being sleeping under an ITN, followed by using mosquito repellants and regularly doing clean-ups around the house, in accordance with records in Kenya [41] and Zimbabwe [42]. The other preventive measures such as wearing protective clothing, using insecticide spray, getting rid of stagnant water, clearing bushes around the house, and using mosquito barrier nets on windows and doors, etc. mentioned by caregivers as good and effective strategies for vector control are in-line with previous study done by Gabriel et al [43].

A good anti-malaria practice is also supported by previous studies [44, 45], which showed $77 \%$ caregivers did come to the hospital first when their child(ren) had a fever. From the epidemiological and clinical perspective, fever is not only a crucial indicator for malaria especially in children, but even the most important warning indicator for administered treatment as recommended by WHO and the Sierra Leone NMCP, especially for the government to offer free health care services for children under-5 [46]. However, some caregivers still preferred the priority of going to a pharmacy or self-medicating when their child(ren) presented fever, which is a dangerous practice that predisposes the sick child(ren) to possibly worse situations. Other studies in Uganda [47] and Myanmar [48] have also found poor care-seeking behaviors for fever cases in children under-5. In our study, furthermore, more than $93 \%$ of caregivers were influenced to seek appropriate medical care by the conditions of their children, and some of the caregivers also mentioned during further explanations that they would decide to go with their child(ren) to the hospital 
only if they saw no improvement after self-medicating and observation for a few days. A similar finding was observed in a study done in Sudan where some parents postponed seeking medical care for their child(ren) for a few days so that the child(ren)'s conditions ran deteriorated by the time to go to the medical facilities [49]. Our findings showed, nevertheless, about $54.6 \%$ of caregivers did seek appropriate medical care within 24 hours for their feverish child(ren). As follow-up therapeutic care, $71.4 \%$ of the caregivers followed and completed a full course of antimalarial medications, which is very important in maximizing antimalarial efficacy with respect to dosing regimens and preventing relapse. But $12.9 \%$ did suspended the administration as soon as their child(ren) showed a little improvement in their clinical symptoms and signs, and surprisingly, some even said they administered the medication to other siblings, all of which are considered harmful practices. Another critical point worth our attention is that previous studies [50] have confirmed resistance to ACTs in some parts of the world like south-east Asia, and they have also pointed out that the reduced efficacy has raised major concerns about malaria treatment and control. For this reason, it is essential to follow correct dosing regimens as prescribed in order to limit the spread of antimalarial resistance.

\section{Correlation among malaria related KAPs}

Although some studies found that high knowledge about prevention was poorly reflected in practice [8, 29], some other studies [30,31] found that, high or increased knowledge about malaria and its mode of transmission and infection could promote and improve preventative practices in communities where malaria is highly prevalent with malaria. Our findings indicated that good knowledge on malaria might lead to positive attitudes, and better attitudes might facilitate good practices against malaria.

\section{Correlation between caregivers' socio-demographic characteristics and KAPs}

The majority of the caregivers of children under- 5 in this study were young mothers ( $\nabla 30$ year-old), and $27.1 \%$ of them lacked any educational experience. Financially, most of them were unemployed and underprivileged: even among the $44.3 \%$ employed caregivers, some were self-employed, mostly engaged in petty trading and low income businesses from which they could hardly earn enough to meet their basic daily needs. And more importantly, 33 younger students among them revealed the existence of early child bearing mothers not well matured to take up the responsibility of motherhood lacking the amount of time and resources for care to their child(ren). For a relatively immature parent that is socioeconomically handicapped, having the right malaria related KAPs might make up for their deficiency in maturity and their socioeconomic disadvantages [18]. But being a mother does have more positive attitudes and practices towards malaria prevention and control than a father, which supports why only women were engaged in most previously conducted studies on malaria perception or prevention and attitudes $[19,20]$. Meanwhile, having at least secondary level of education was found associated with a positive influence on malaria related attitude and knowledge, which is consistent with previous studies showing the importance of education in caregivers [7, 21]. But Nzooma et al. [11] in Zambia reported that higher levels of education were not related to higher knowledge levels and good practices towards malaria control. Our findings indicated that the student mothers might know more but do less. 
Moreover, across the surveyed groups: urban-based caregivers and rural-based caregivers, only $1.2 \%$ of urban-based caregivers expressed their unawareness of the lethality of the disease whereas the number for rural-based caregivers was over four times higher (5.6\%). It is interesting to note that significantly more urban-based caregivers (79.8\%) followed a complete malaria treatment course of ACTs than the rural (63.3\%). But in view of using ITNs for malaria prevention, the rural-based caregivers $(85.3 \%)$ presented significantly more positive attitudes than the urban (69.9\%), and the rural $(70.1 \%)$, therefore, represented better practices than the urban (52.0\%).

\section{Limitations of this study}

The present study is a cross-sectional survey which can benefit the understanding of the KAPs towards malaria, but it does not provide information about the cause and effect of malaria in children under-5. On the other hand, across the whole survey the respondents' answers were self-reported. Consequently, there may have been limitations in the provisions of exact and specific information by the caregivers and the possibility of interviewer bias, participant bias or response bias.

\section{Conclusions}

The present KAPs survey towards malaria prevention and control indicated that misconception of the cause, transmission and clinical symptoms, unawareness of the lethality and severity, and inappropriate behaviors in prevention and treatment including self-medicating are still in existence among the caregivers of children under-5 in the Western Area of Sierra Leone. The KAPs towards malaria were affected by the caregivers' educational level, occupational status, motherhood/fatherhood, living in rural/urban region, and religion. Positive correlations in knowledge-attitudes and attitudes-practices were demonstrated. Therefore, in order to better protect children under- 5 against severe and deadly malaria, their caregivers, especially young mothers including single student moms, need priority support. More attention should be given to develop efficient and accurate message transmission system towards malaria prevention and control including the content, type, and media, especially strengthening community-based media infrastructure such as the internet, social media, TV, and radio, etc. It should be highly considered as well to create more education and employment opportunities for women of childbearing age. Meanwhile it is worthwhile to set up the community-based counselling services towards malaria, especially for caregivers of children under-5. Since the WHO's report highlighted Sierra Lenone's heavy malaria burden, the government's creation of the NMCP has brought the country's rural and urban population in-line with other endemic countries in terms of malaria control. The present study has allowed us to focus our efforts on the predominant causes, which may facilitate to improve implementation of integrated malaria control strategies, special for dealing with infections in children under-5.

\section{Abbreviations}


KAPs: Knowledge, Attitudes and Practices; WAR: Western Area Rural; WAU: Western Area Urban; ACTs: Artemisinin-based Combination Therapies; SSA: Sub-Saharan Africa; NMCP: National Malaria Control Programme; SLMIS: Sierra Leone Malaria Indicator Survey; WHO: World Health Organization; CDC: Center for Disease Control and Prevention; HIV/AIDS: Human Immunodeficiency Virus/ Acquired Immunodeficiency Syndrome; TB: Tuberculosis; ITNs: Insecticides Treated Nets; SLDHS: Sierra Leone Demographic Health Survey; IPT: Intermittent Preventive Treatment; SDGs: Sustainable Development Goals;.

\section{Declarations}

\section{Ethical approval and consent to participate}

Ethical and scientific clearance was sought and obtained from the Sierra Leone Ethics and Scientific Review Committee. Also, the approval and permission of the medical director, superintendent or hospital manager of the various selected hospitals were sought and obtained for this study. Data collection was done using the study questionnaire and by a face-to-face interview method. After gaining ethical clearance and permission from the respective authorities to carry on with the study, caregivers who visited the out-patient department of the selected hospitals and met the inclusion criteria were approached and asked for their consent to partake in the study. The purpose of the study was explained to them and those who gave their consent to take part did so by signing or thumbrinting the informed consent and were assured of confidentiality before proceeding.

\section{Consent for publication}

Not applicable

\section{Availability of data and materials}

The datasets produced by the current study is available from the corresponding author upon reasonable request.

\section{Competing interests}

The authors declare that they have no competing interests.

\section{Funding}

This study was supported by National Key R\&D Program of China (2020YFC1200100), the Guangzhou Synergy Innovation Key Programme for Health (201803040006), National Natural Science Foundation of China (82072311).

\section{Authors' contributions}


Conceptualization and design: XHZ. Questionnaire interview and data collection: JMK. Data cleaning: JMK, JJKN and AMEAG. Data analysis and visualization: YJW, JMK, XG, LL, XQZ and ZYL. Interpretation of results: XHZ, JMK, and YJW. Writing-original draft: JMK and YJW. Writing-review \& editing: XHZ and RKW. All authors read and approved the final manuscript.

\section{Acknowledgements}

We would like to acknowledge the Sierra Leone Ethics and Scientific Review Committee for their support for this study to be carried out in the Freetown, Sierra Leone. We also thank the participants of this study for their time and cooperation.

\section{References}

1. (WHO) WHO. WHO World Malaria Report 2019. 2019.

2. DePina AJ, Dia AK, de Ascencao Soares Martins A, Ferreira MC, Moreira AL, Leal SV, et al. Knowledge, attitudes and practices about malaria in Cabo Verde: a country in the pre-elimination context. BMC Public Health. 2019; 19:850.

3. Government of Sierra Leone Ministry of Health and Sanitation National Malaria Control Programme. Guidelines for case management of malaria in Sierra Leone. Fourth edition; WHO Africa: Publications; 2015.

4. NMCP, INFORM and LSHTM. Sierra Leone: A profile of malaria control and epidemiology. 2015.

5. Tabbabi A. Socio-economic impact of malaria in Africa. Acta scientific microbiology. 2018; 1:32-34.

6. Government of Sierra Leone Ministry of Health and Sanitation. Sierra Leone Malaria Control Strategic Plan 2016-2020; 2015.

7. Oladimeji KE, Tsoka-Gwegweni JM, Ojewole E and Yunga ST. Knowledge of malaria prevention among pregnant women and non-pregnant mothers of children aged under 5 years in Ibadan, South West Nigeria. Malar J. 2019; 18:92.

8. Hlongwana KW, Mabaso ML, Kunene S, Govender D and Maharaj R. Community knowledge, attitudes and practices (KAPs) on malaria in Swaziland: a country earmarked for malaria elimination. Malar J. 2009; 8:29.

9. Khairy S, Al-Surimi K, Ali A, Shubily HM, Al Walaan N, Househ M, et al. Knowledge, attitude and practice about malaria in south-western Saudi Arabia: A household-based cross-sectional survey. J Infect Public Health. 2017; 10:499-506.

10. Yaya S, Bishwajit G, Ekholuenetale M, Shah V, Kadio B and Udenigwe O. Knowledge of prevention, cause, symptom and practices of malaria among women in Burkina Faso. PLoS One. 2017; 12:e0180508.

11. Shimaponda-Mataa NM, Tembo-Mwase E, Gebreslasie M and Mukaratirwa S. Knowledge, attitudes and practices in the control and prevention of malaria in four endemic provinces of Zambia. Southern African Journal of Infectious Diseases. 2016; 32:29-39. 
12. Kassam R, Sekiwunga R, MacLeod D, Tembe J and Liow E. Patterns of treatment-seeking behaviors among caregivers of febrile young children: a Ugandan multiple case study. BMC Public Health. 2016; 16:160.

13. Kadam P and Bhalerao S. Sample size calculation. Int J Ayurveda Res. 2010; 1:55-57.

14. S. PRESIDENT'S MALARIA INITIATIVE. Sierra Leone malaria operational plan. FY 2018 \& FY 2019; 2020.

15. Gupta RK, Raina SK, Shora TN, Jan R, Sharma R and Hussain S. A household survey to assess community knowledge, attitude and practices on malaria in a rural population of Northern India. Journal of family medicine and primary care. 2016; 5:101-107.

16. Janet K, Catherine A, Folake O, Precious $O$ and Yewande DA. Knowledge, Attitudes and Practices of Mothers of Under-Five Regarding Prevention of Malaria in Children: Evidence from ogun State, Nigeria. IOSR Journal of Humanities and Social Science. 2016; 21:01-07.

17. Cohen J. Statistical Power Analysis for the Behavioral Sciences. Second edition. United States of America: Lawrence Erlbaum Associates; 1988. 567 p.

18. Ricci F. Social implications of malaria and their relationships with poverty. Mediterr J Hematol Infect Dis. 2012; 4:e2012048.

19. Davies M. Womens Perceptions of Malaria in the Western Rural Areas of Sierra Leone. Walden University; 2018.

20. Orimadegun AE and llesanmi KS. Mothers' understanding of childhood malaria and practices in rural communities of Ise-Orun, Nigeria: implications for malaria control. J Family Med Prim Care. 2015; 4:226-231.

21. Mitiku I and Assefa A. Caregivers' perception of malaria and treatment-seeking behaviour for under five children in Mandura District, West Ethiopia: a cross-sectional study. Malar J. 2017; 16:144.

22. Mazigo HD, Obasy E, Mauka W, Manyiri P, Zinga M, Kweka EJ, et al. Knowledge, Attitudes, and Practices about Malaria and Its Control in Rural Northwest Tanzania. Malar Res Treat. 2010; 2010:794261.

23. Smithson P, Florey L, Salgado SR, Hershey CL, Masanja H, Bhattarai A, et al. Impact of Malaria Control on Mortality and Anemia among Tanzanian Children Less than Five Years of Age, 1999-2010. PLoS One. 2015; 10:e0141112.

24. Nejati J, Moosa-Kazemi SH and Saghafipour A. Soofi K Knowledge, attitude and practice (KAPs) on malaria, from high malaria burden rural communities, southeastern Iran. J Parasit Dis. 2018; 42:6267.

25. Ahmed SM, Haque R, Haque $U$ and Hossain A. Knowledge on the transmission, prevention and treatment of malaria among two endemic populations of Bangladesh and their health-seeking behaviour. Malar J. 2009; 8:173.

26. Govere J, Durrheim D, la Grange K, Mabuza A and Booman M. Community knowledge and perceptions about malaria and practices influencing malaria control in Mpumalanga Province, South Africa. S Afr Med J. 2000; 90:611-8. 
27. Obol J, David Lagoro K and Garimoi OC. Knowledge and Misconceptions about Malaria among Pregnant Women in a Post-Conflict Internally Displaced Persons' Camps in Gulu District, Northern Uganda. Malar Res Treat. 2011; 2011:107987-107994.

28. Mathania MM, Kimera SI and Silayo RS. Knowledge and awareness of malaria and mosquito biting behaviour in selected sites within Morogoro and Dodoma regions Tanzania. Malar J. 2016; 15:287.

29. Singh RK, Haq S and Dhiman RC. Studies on knowledge, attitude and practices in malaria endemic tribal areas of Bihar and Jharkhand, India. J Trop Dis Public Health. 2013; 1:110.

30. Israel OK, Fawole OI, Adebowale AS, Ajayi IO, Yusuf OB, Oladimeji A, et al. Caregivers' knowledge and utilization of long-lasting insecticidal nets among under-five children in Osun State, Southwest, Nigeria. Malar J. 2018; 17:231.

31. Eseigbe EE, Anyiam JO, Ogunrinde GO, Wammanda RD and Zoaka HA. Health Care Seeking Behavior among Caregivers of Sick Children Who Had Cerebral Malaria in Northwestern Nigeria. Malar Res Treat. 2012; 2012:954975.

32. Dujing SL. Malaria in children under 5 yearsin the Gushegu district hospital before and after indoor residual spraying in northern Ghana. College of health sciences university of Ghana, School of public health, 2015.

33. Nyirakanani C, Chibvongodze R, Habtu M, Masika M, Mukoko D and Njunwa KJ. Prevalence and risk factors of asymptomatic malaria among under-five children in Huye District, Southern Rwanda. Tanzania J Health Res. 2018; 20(1):1-7.

34. Statistics Sierra Leone (SSL) and ICF International. Sierra Leone Demographic and Health Survey 2013. Freetown, Sierra Leone and Rockville, Maryland, USA: SSL and ICF International; 2014

35. Htwe EP. Caregivers' Malaria Preventive Practices for Under-Five Children and its association in Ngapudaw high-risk township, Ayeyarwady region-Myanmar. Chulalongkorn University; 2017.

36. Lungu EA, Obse AG, Darker $C$ and Biesma R. What influences where they seek care? Caregivers' preferences for under-five child healthcare services in urban slums of Malawi: A discrete choice experiment. PLoS One. 2018; 13:e0189940.

37. O'Donnell AJ, Rund SSC and Reece SE. Time-of-day of blood-feeding: effects on mosquito life history and malaria transmission. Parasit Vectors. 2019; 12:301.

38. Pigeault R, Caudron Q, Nicot A, Rivero A and Gandon S. Timing malaria transmission with mosquito fluctuations. Evol Lett. 2018; 2:378-389.

39. Guidelines for the treatment of malaria-3rd edition. Switzerland: Global malaria programme; 2015.

40. National Malaria Control Programme, Statistics Sierra Leone, University of Sierra Leone, Catholic Relief Services, and ICF. Sierra Leone Malaria Indicator Survey 2016. Freetown, Sierra Leone: NMCP, SSL, CRS, and ICF; 2016.

41. Taylor C, Florey L and Ye Y. Equity trends in ownership of insecticide-treated nets in 19 sub-Saharan African countries. Bull World Health Organ. 2017; 95:322-332. 
42. Tizifa TA, Kabaghe AN, McCann RS, van den Berg H, Van Vugt M and Phiri KS. Prevention Efforts for Malaria. Curr Trop Med Rep. 2018; 5:41-50.

43. Gachelin G, Garner P, Ferroni E, Verhave JP and Opinel A. Evidence and strategies for malaria prevention and control: a historical analysis. Malar J. 2018; 17:96.

44. Mpimbaza A, Ndeezi G, Katahoire A, Rosenthal PJ and Karamagi C. Demographic, Socioeconomic, and Geographic Factors Leading to Severe Malaria and Delayed Care Seeking in Ugandan Children: A Case-Control Study. Am J Trop Med Hyg. 2017; 97:1513-1523.

45. Adinan J, Damian DJ, Mosha NR, Mboya IB, Mamseri R and Msuya SE. Individual and contextual factors associated with appropriate healthcare seeking behavior among febrile children in Tanzania. PLoS One. 2017; 12:e0175446.

46. MEASURE Evaluation. Capacity of Sierra Leone's National Malaria Control Programme for Monitoring and Evaluation: Baseline Assessment. Chapel Hill, NC, USA: MEASURE Evaluation, University of North Carolina; 2019.

47. Mpimbaza A, Ndeezi G, Katahoire A, Rosenthal PJ and Karamagi C. Demographic, Socioeconomic, and Geographic Factors Leading to Severe Malaria and Delayed Care Seeking in Ugandan Children: A Case-Control Study. Am J Trop Med Hyg. 2017; 97:1513-1523.

48. Thandar MM, Kyaw MP, Jimba M and Yasuoka J. Caregivers' treatment-seeking behaviour for children under age five in malaria-endemic areas of rural Myanmar: a cross-sectional study. Malaria J. 2015; 14:1.

49. Elfaki AEM, Elnimeiri MKM and Elfakey WEM. Management seeking behavior of malaria among mothers of under-five year's children in Damazin Locality, Blue Nile State, Sudan. International Journal of Pharmaceutical Science Invention. 2017; 6(10):14-19.

50. Beeson JG, Boeuf P and Fowkes FJ. Maximizing antimalarial efficacy and the importance of dosing strategies. BMC Med. 2015; 13:110.

\section{Tables}

Due to technical limitations, table 1, 2, 3 and 4 is only available as a download in the Supplemental Files section.

\section{Figures}




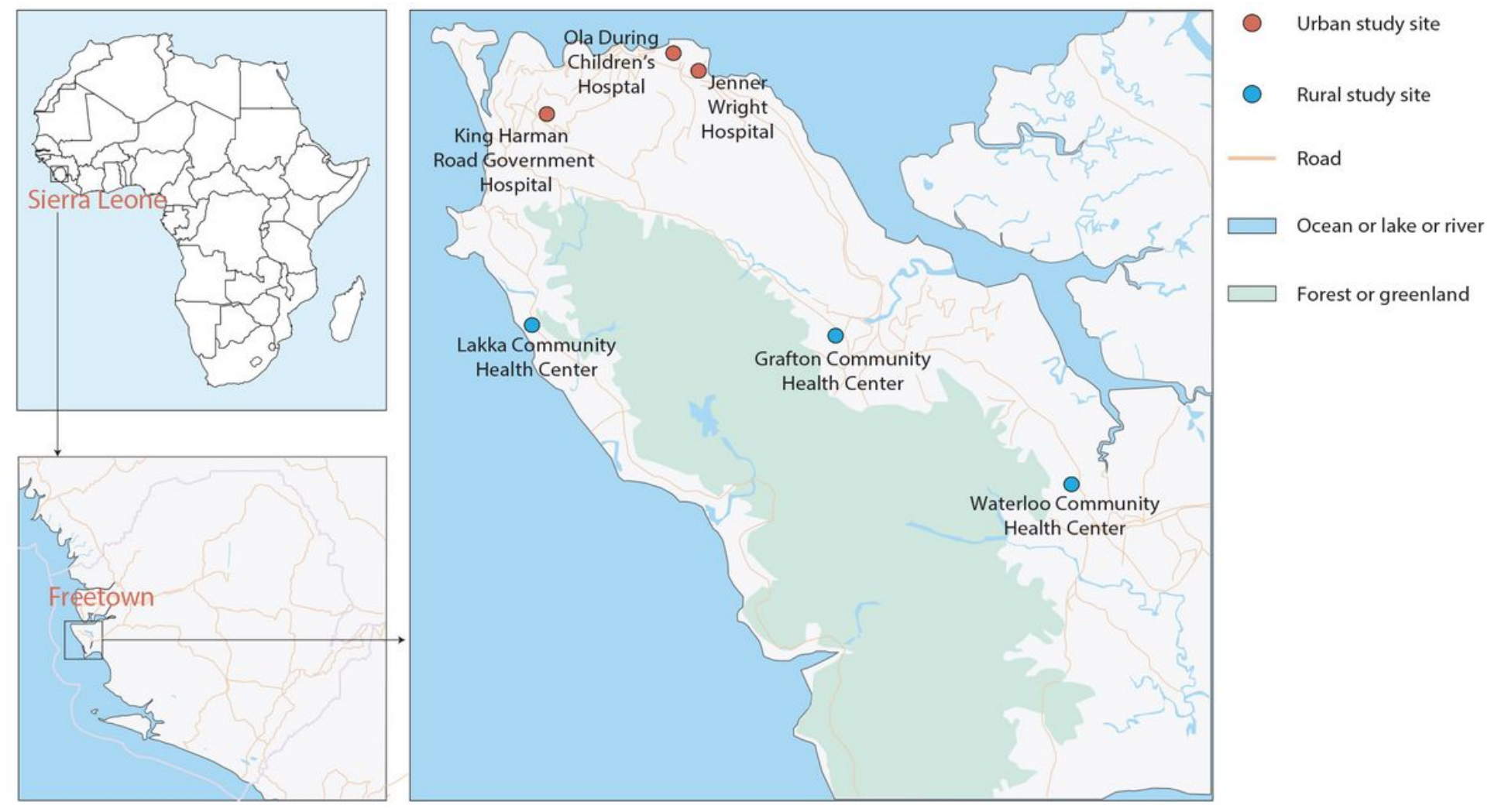

\section{Figure 1}

Geographic location of the study sites. Health care facilities in WAU included: Ola During Children's Hospital (ODCH) (8029'25"E, 13o13'7"S), King Harmann Road Government Hospital (8o28'27"E, 13o14'52"S), and Jenner Wright Hospital (8029'7"E, 13012'43"S). Health care facilities in WAR included: Waterloo Community Health Center (8020'08"E, 13o4'24"S), Grafton Community Health Center (8023'34"E, 1309'23"S), and Lakka Community Health Center (8023'49"E, 13o15'50"S). Note: The designations employed and the presentation of the material on this map do not imply the expression of any opinion whatsoever on the part of Research Square concerning the legal status of any country, territory, city or area or of its authorities, or concerning the delimitation of its frontiers or boundaries. This map has been provided by the authors. 


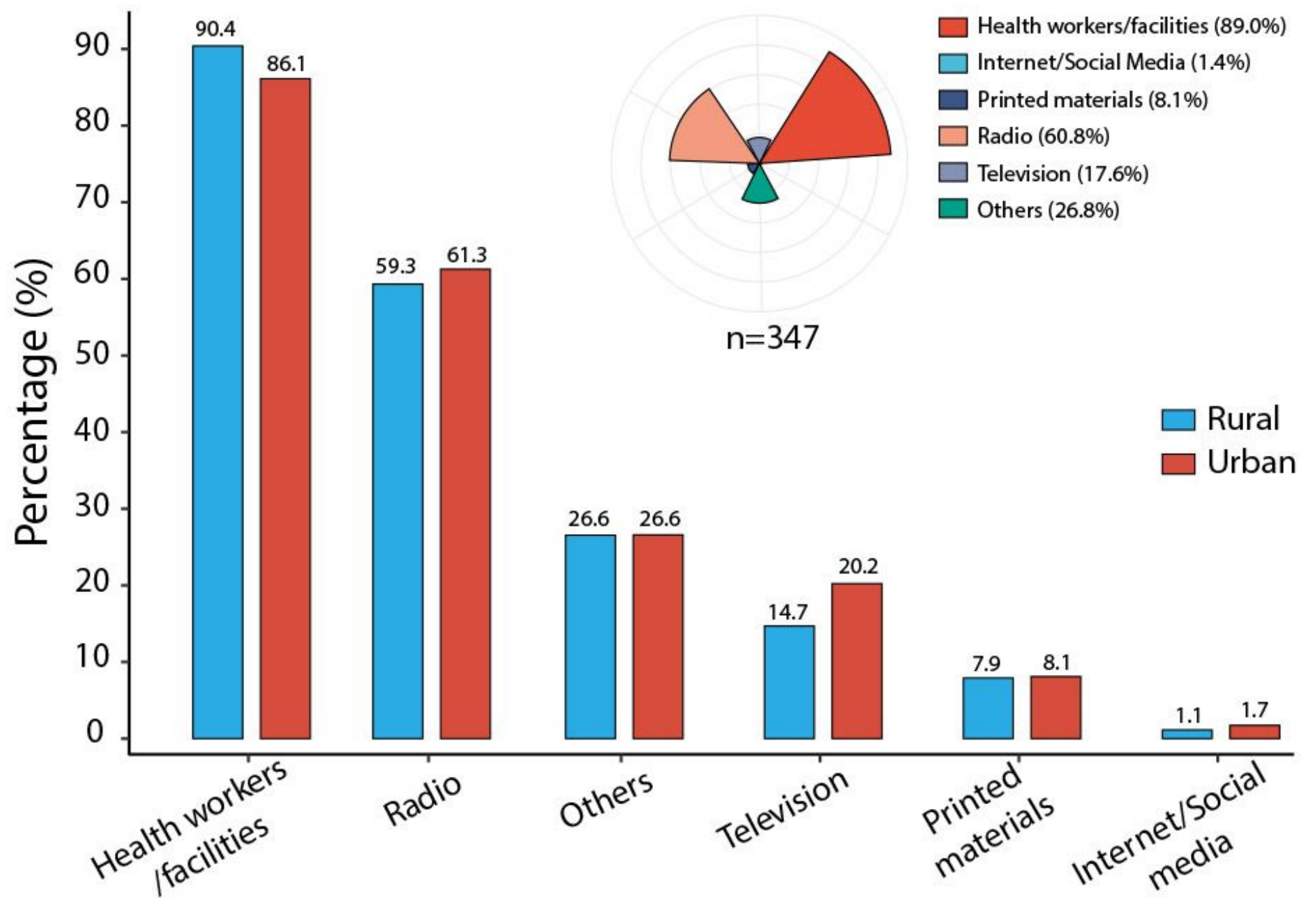

Figure 2

Sources of malaria related information mentioned by caregivers of children under- 5 . The upper right rose diagram shows the proportion of each source of malaria related information selected by the caregivers in this study, the different colors represent different sources. The bar plot shows the popularizing rate of the specific source across rural and urban districts, blue and red represent the rural and urban, respectively. 


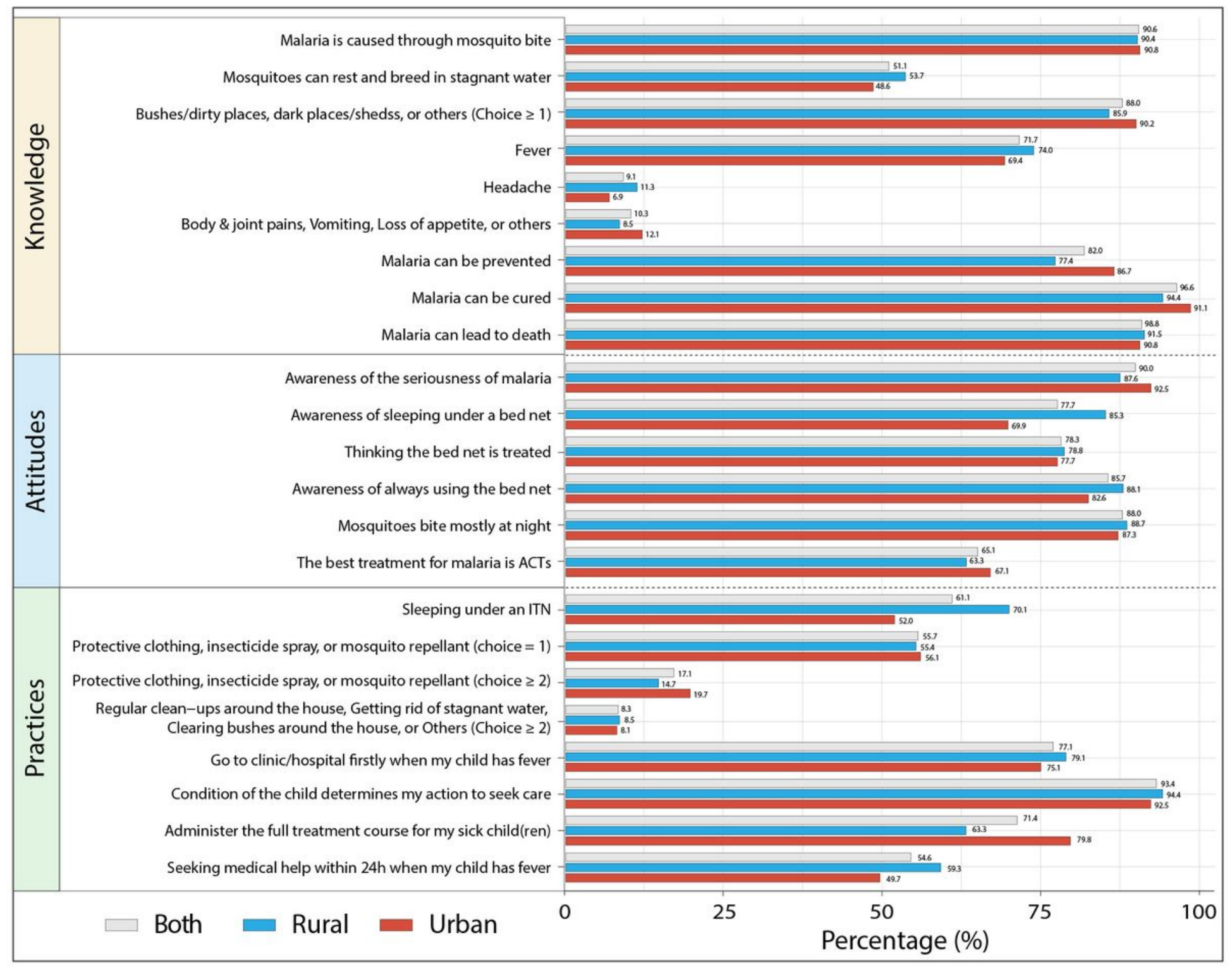

\section{Figure 3}

Correct response rates for questions in sections-Knowledge, Attitudes, and Practices. Each item scores 1, except for "Sleeping under an ITN" in section Practices scoring 2, which were evaluated by the scoring system described in Additional file 2. 

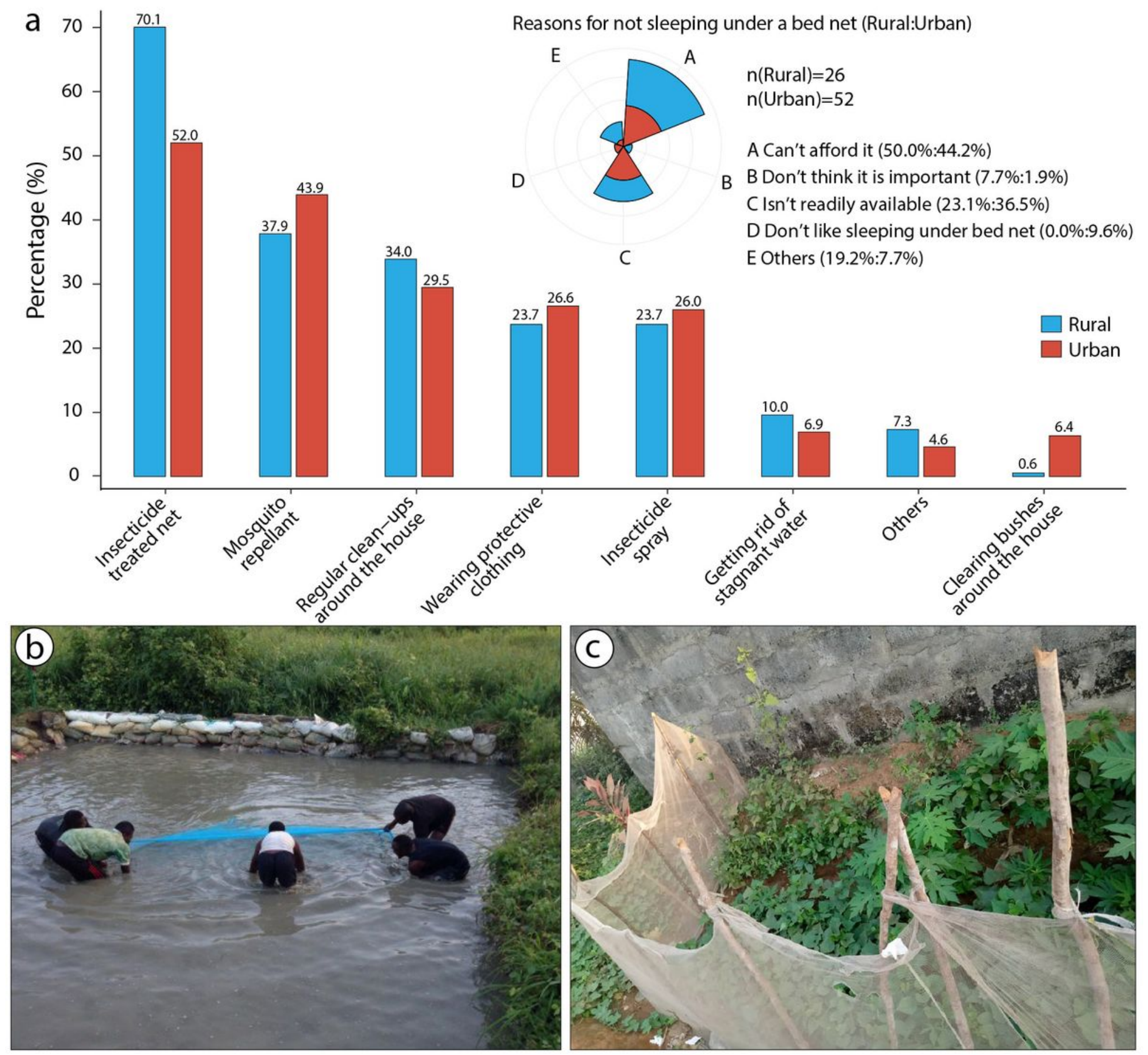

Figure 4

a: Protective measures against mosquito bites and the reasons for not sleeping under a bed net. The upper right rose diagram shows the reasons why some caregivers did not use ITNs in both rural and urban districts, and the proportion of the reason is shown behind the legend as Rural : Urban (\%). The bar plot shows the protective measures against mosquito bites, employed by caregivers of children under- 5 in rural and urban districts. Blue and red represent the rural and urban, respectively. b and c: Images of mosquito bed net misuse in Sierra Leone. b: Some individuals were doing pond fishing using mosquito bed nets. c: Small backyard gardens were covered with mosquito bed nets. Misuse of the bed nets like these is common especially in rural and provincial areas of Sierra Leone. 


\section{Supplementary Files}

This is a list of supplementary files associated with this preprint. Click to download.

- Table1.pdf

- Table2.pdf

- Table3.pdf

- Table4.pdf

- Additionalfile1.pdf

- Additionalfile2.pdf

- Additionalfile3.pdf

- Additionalfile4.pdf

- Additionalfile5.pdf 